\title{
Evaluation of the Hemodynamic Relationship Between the Left Atrium and Left Ventricle During Atrial Systole by Pulsed Tissue Doppler Imaging in Patients With Left Heart Failure
}

\author{
Miho Abe, MD; Takashi Oki, MD; Tomotsugu Tabata, MD; \\ Hirotsugu Yamada, MD; Yukiko Onose, MD; Masako Matsuoka, MD; \\ Yuichiro Mishiro, MD; Tetsuzo Wakatsuki, MD; Susumu Ito, MD
}

\begin{abstract}
The objective of the present study was to evaluate the hemodynamic relationship between the left atrium (LA) and left ventricle (LV) during atrial systole in the presence of an elevated left ventricular end-diastolic pressure (LVEDP) and LV failure using pulsed tissue Doppler imaging (TDI). Fifty-three patients with LV systolic dysfunction and no regional LV asynergy were divided into 3 groups: relaxation failure group $(R F, n=20)$ with a ratio of peak early diastolic to atrial systolic velocity of the transmitral flow $(\mathrm{E} / \mathrm{A}) \leq 1$; pseudonormalization group (PN, n=19) with $1<\mathrm{E} / \mathrm{A}<2$; and restrictive group (RS, n=14) with $\mathrm{E} / \mathrm{A} \geq 2$. In addition, 20 normal patients $(\mathrm{E} / \mathrm{A} \geq 1)$ were studied as a control group. The transmitral and pulmonary venous flow velocities were recorded by transesophageal pulsed Doppler echocardiography. The wall motion velocity patterns were recorded at the middle portion of the LV posterior wall (LVPW) and at the mitral annulus (MA) of the LVPW site in the apical LV long-axis view by transthoracic pulsed TDI. The LVEDP was significantly greater in the PN and RS groups than in the RF and control groups. The mean pulmonary capillary wedge pressure was greatest in the RS group. The percent fractional change of the LA area during atrial systole determined by 2-dimensional echocardiography was significantly lower in the RS group than in the PN group. The peak atrial systolic pulmonary venous flow velocity was significantly greater in the PN group than in the RS group. The peak atrial systolic motion velocity (Aw) at the LVPW was significantly lower in the PN and RS groups than in the RF and control groups. The Aw at the MA was significantly lower in the RS group than in the other groups. There was no significant difference in Aw between the LVPW and MA in the RS group, whereas Aw at the MA was significantly greater than that at the LVPW in the PN group. In conclusion, the measurements of Aw at the LVPW and MA can be used to noninvasively evaluate the hemodynamic relationship between the LA and LV during atrial systole in patients with LV failure. (Jpn Circ J 1999; 63: 763-769)
\end{abstract}

Key Words: Atrial systolic wall motion velocity; Left ventricular end-diastolic pressure; Left ventricular posterior wall; Mean pulmonary capillary wedge pressure; Mitral annulus; Pulsed tissue Doppler imaging

$\mathbf{M}$ easurements obtained from the transmitral flow (TMF) velocity pattern are used most frequently for evaluating left ventricular (LV) diastolic function noninvasively 1,2 However, the TMF velocity is influenced by loading conditions, especially preload, ${ }^{3-6}$ as exemplified by the 'pseudonormalization' (PN) or 'restrictive' (RS) pattern of the TMF velocity in the presence of an elevated LV end-diastolic pressure (LVEDP)! Accordingly, detailed information regarding the LV filling hemodynamics can be obtained by evaluating pulmonary venous flow (PVF) velocity patterns together with TMF velocity patterns in patients with an elevated LVEDP ${ }^{7-9}$ On the other hand, LV function along the long axis can be evaluated by the mitral annular motion recorded by M-mode echocardiography, ${ }^{10-13}$ and it is expected that unique infor-

(Received April 30, 1999; revised manuscript received July 7, 1999; accepted July 9, 1999)

The Second Department of Internal Medicine, School of Medicine, The University of Tokushima, Tokushima, Japan

Mailing address: Takashi Oki, MD, The Second Department of Internal Medicine, School of Medicine, The University of Tokushima, 2-50 Kuramoto-cho, Tokushima 770-8503, Japan mation may be obtained by assessing mitral annular motion. Tissue Doppler imaging (TDI) involves the selective display of large Doppler signals obtained from the ventricular wall as a color or pulsed Doppler image through the elimination of small Doppler signals produced by blood flow!4-16 Atrial systolic waves from the LV posterior wall (LVPW) motion velocity patterns obtained by pulsed TDI are generated by blood entering the LV following left atrial (LA) contraction!7,18 In contrast, the atrial systolic waves from the mitral annular motion velocity patterns are believed to be influenced by abnormal hemodynamic events occurring not only on the LV side but also on the LA side!1,12,19,20 Therefore, both motion velocity patterns may vary depending on the hemodynamic conditions in the LA and LV in patients with LV systolic dysfunction. The present study investigated LVPW motion velocity patterns and mitral annular motion velocity patterns at the LVPW site in the apical LV long-axis view by pulsed TDI in patients with LV failure, and determined the hemodynamic relationship between the LA and LV during atrial systole in the presence of an elevated LVEDP and LV failure. 
TMF
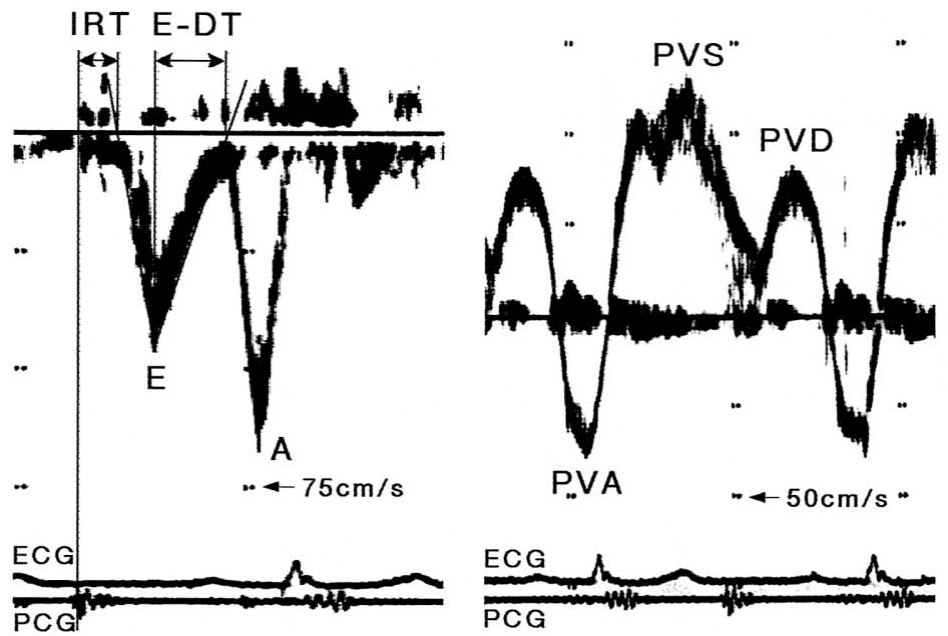

Methods

\section{Study Population}

Of 650 consecutive patients who underwent echocardiography and cardiac catheterization between April 1996 and March 1998, we enrolled 53 patients in sinus rhythm who met the following inclusion criteria: (1) transesophageal echocardiography and TDI were performed within $3 \mathrm{~h}$ before cardiac catheterization, (2) the LV ejection fraction (LVEF) determined by left ventriculography was $<40 \%$ and/or the percent LV fractional shortening determined by M-mode echocardiography was $<25 \%$, (3) there was no evidence of regional LV asynergy, and (4) there was no evidence of moderate or severe valvular heart diseases.

The 53 patients included 30 patients with dilated cardiomyopathy (mean age: $52 \pm 18$ years, range: $28-79$ years), 16 patients with ischemic cardiomyopathy 21 (mean age: $59 \pm 11$ years, range: $45-79$ years), and 7 patients with cardiac amyloidosis (mean age: $56 \pm 17$ years, range: $34-77$ years). Twenty patients, who had been admitted with the chief complaints of chest pain, palpitations, dyspnea or heart murmur, were included as controls. Phonocardiography, routine echocardiography and cardiac catheterization did not demonstrate organic cardiac disease, and none of the 20 patients had a systemic disease such as diabetes mellitus. To compare with the PN or RS patterns of the TMF velocity in the disease groups, our selected 20 patients had a ratio of early diastolic to atrial systolic TMF velocity $(E / A) \geq 1$. Therefore, the mean age of the control group (mean age: $38 \pm 8$ years, range: $27-56$ years) was lower than that in the other groups. The purpose of this study was fully explained to all patients, and informed consent was obtained.

\section{M-Mode and Two-Dimensional Echocardiography}

Transthoracic M-mode echocardiograms were recorded with a commercially available Toshiba SSA-380A (Toshiba Corp, Tokyo, Japan) ultrasound diagnostic system using a $2.5 \mathrm{MHz}$ probe. The maximum LA dimension, $\mathrm{LV}$ enddiastolic dimension (LVDd), LV end-systolic dimension (LVDs), end-diastolic ventricular septal thickness (VSth), and end-diastolic LVPW thickness (PWth) were determined. The percent LV fractional shortening (\%FS) and
Fig 1. Parameters from transmitral flow (TMF) and pulmonary venous flow (PVF) velocities recorded by transesophageal pulsed Doppler echocardiography. E, peak early diastolic velocity of TMF; A, peak atrial systolic velocity of TMF; IIA, aortic component of the second heart sound; IRT, isovolumic relaxation time; E-DT, deceleration time from the peak early diastolic velocity of TMF to the baseline; PVS, peak second systolic velocity of PVF; PVD, peak early diastolic velocity of PVF; PVA, peak atrial systolic velocity of PVF; ECG, electrocardiogram; PCG, phonocardiogram. mean LV wall thickness (mLVth) at end-diastole were calculated using the following equations, respectively:

$$
\begin{aligned}
\% \mathrm{FS} & =[(\mathrm{LVDd}-\mathrm{LVDs}) / \mathrm{LVDd}] \times 100 \\
\mathrm{mLVth} & =(\mathrm{VSth}+\mathrm{PWth}) / 2
\end{aligned}
$$

Transthoracic 2-dimensional apical 4-chamber echocardiograms were also recorded with the same ultrasound diagnostic system using a $2.5 \mathrm{MHz}$ probe. The percent fractional change of the LA area during atrial systole (LA$\% \mathrm{AC}$ ) was calculated with the following equation:

$$
\begin{aligned}
\mathrm{LA}-\% \mathrm{AC}= & {[(\text { pre-LA area }- \text { post-LA area })} \\
& / \text { pre-LA area }] \times 100
\end{aligned}
$$

in which the pre-LA area and post-LA area are the LA area immediately before and after atrial contraction, respectively.

\section{Transesophageal Echocardiography}

Transesophageal echocardiography was performed using a commercially available Aloka SSD 870 (Aloka Co, Ltd, Tokyo, Japan) ultrasound diagnostic system with a 5.0 $\mathrm{MHz}$ biplane probe. The sample volume was placed at the tip of the mitral valve leaflets in the longitudinal image including the LA and LV, and the TMF velocity was recorded using pulsed Doppler method. The peak early diastolic velocity $(\mathrm{E})$, the peak atrial systolic velocity $(\mathrm{A})$, and their ratio (E/A), the deceleration time of the early diastolic TMF velocity (E-DT), and the time between the aortic component of the second heart sound and the onset of the early diastolic wave (IRT) were determined (Fig 1, TMF). The 53 patients were divided into 3 groups: the relaxation failure group ( $\mathrm{RF}, \mathrm{n}=20$ ) with an $\mathrm{E} / \mathrm{A} \leq 1$ (mean age: $55 \pm 15$ years, range: $29-71$ years), the PN group $(n=19)$ with an E/A between 1 and 2 (mean age: $57 \pm 16$ years, range: $28-75$ years), and the RS group $(n=14)$ with an $\mathrm{E} / \mathrm{A} \geq 2$ (mean age: $53 \pm 17$ years, range: $27-65$ years).

The PVF velocity pattern was recorded at the level of the horizontal section of the LA that included the left superior pulmonary vein, using the pulsed Doppler method. The sample volume was placed within $1-2 \mathrm{~cm}$ of the junction of the pulmonary vein with the LA. Based on the PVF velocity patterns obtained, the peak second systolic velocity (PVS), the peak early diastolic velocity (PVD), and the peak atrial 
systolic velocity (PVA) were determined (Fig 1, PVF).

\section{Tissue Doppler Imaging}

Tissue Doppler imaging was performed using a commercially available Toshiba SSA-380A (Toshiba Corp) ultrasound diagnostic system with a $3.5 \mathrm{MHz}$ probe. Using the apical long-axis view of the LV, the sample volumes were set at the endocardial portion of the middle site of the LVPW and at the mitral annulus of the LVPW site, and the wall motion velocities were recorded using the pulsed Doppler method (Fig 2, left). Based on the wall motion velocity patterns obtained, the peak early diastolic motion velocities (Ews) and the peak atrial systolic motion velocities (Aws) for the LVPW and the mitral annulus of the LVPW site were determined (Fig 2, right).

All M-mode and pulsed Doppler echocardiographic images were recorded on a strip chart recorder at a paper speed of 50 or $100 \mathrm{~mm} / \mathrm{s}$ along with the electrocardiogram and phonocardiogram. The mean values for 5 consecutive heart beats were used. The interobserver variability of pulsed TDI measurements was calculated as the difference between 2 measurements obtained in the same patient by 2 different observers divided by the mean value (3-9\%). Intraobserver variability also was calculated as the difference between 2 measurements obtained from the same patient by 1 observer divided by the mean value $(2-7 \%)$.

\section{Cardiac Catheterization}

Right heart catheterization was performed within $3 \mathrm{~h}$ after transesophageal echocardiography and pulsed TDI. The pulmonary capillary wedge pressure (PCWP) curve was recorded at end-expiration with the patient in the supine position using a Swan-Ganz catheter (Baxter Healthcare Corp, Santa Ana, CA, USA). A 7F fiberoptic thermodilution catheter was inserted via a subclavian vein puncture and advanced to the distal pulmonary artery. Correct positioning for recording the PCWP was confirmed by demonstrating a clear change in the pressure waveform when the balloon was inflated. Based on the pressure measurements, the mean PCWP (mPCWP) was determined. Fluid-filled lines and Cobe transducers (CD XIII; Cobe Laboratories, Inc, Lakewood, CO, USA) were used for measurements.

The LV pressure curve was recorded on paper at 100 $\mathrm{mm} / \mathrm{s}$ using a $6 \mathrm{~F}$ high-fidelity manometer-tipped catheter inserted via the femoral approach (MICRO-TIP Angiographic catheter Model SPY-464D, Miller Instruments Inc, Houston, TX,USA). The LVEDP was measured just before coronary angiography and left ventriculography. The percent LVEF was also determined during left ventriculography by the area-length method.

\section{Statistical Analysis}

Values are expressed as mean $\pm \mathrm{SD}$. Mean values in different patient groups and at each site of the LVPW and mitral annulus in the same patient were compared by analyses of variance and covariance, respectively, and the Scheffe's F test. A p level $<0.05$ was considered statistically significant.

\section{Results}

\section{Patient Characteristics}

Patient age was significantly lower in the control group than in the RF, PN, and RS groups, although there were no

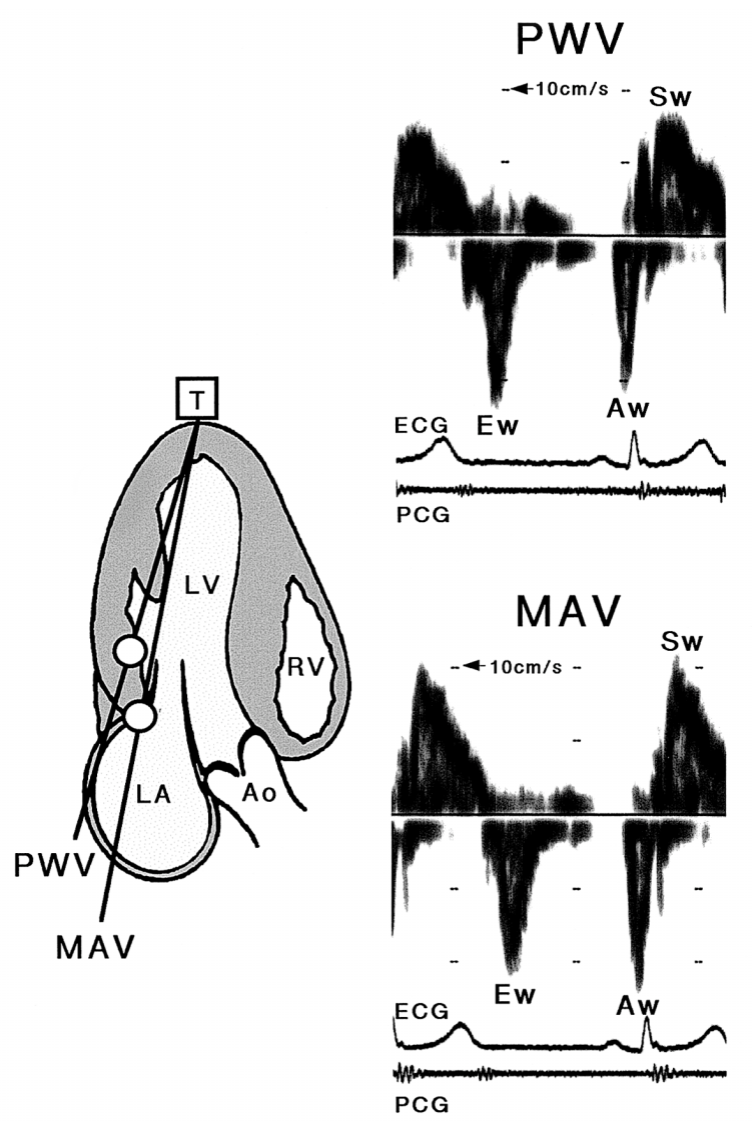

Fig 2. Sample recording of the motion velocity pattern of the left ventricular posterior wall (PWV) and mitral annulus of the PW site (MAV) by pulsed tissue Doppler imaging in the apical long-axis view of the LV (left), and measurement method of PWV and MAV variables (right). T, transducer; RV, right ventricle; LA, left atrium; Ao, ascending aorta; $\mathrm{Sw}$, peak systolic wall motion velocity; Ew, peak early diastolic wall motion velocity; Aw, peak atrial systolic wall motion velocity; ECG, electrocardiogram; PCG, phonocardiogram.

significant differences between the 3 disease groups (Table 1). There were no significant differences in heart rate or systolic blood pressure between the 4 groups. The percent fractional change of the LA area during atrial systole was significantly lower in the RF, PN, and RS groups than in the control group, and significantly lower in the RS group than in the RF and PN groups.

\section{TMF and PVF Variables}

The peak atrial systolic TMF velocity in the RS group was significantly lower than that in the other 3 groups (Table 2). The peak second systolic PVF velocity was significantly lower in the PN and RS groups than in the other 2 groups. However, there were no significant differences in the peak second systolic PVF velocity between the control and RF groups or between the PN and RS groups. The peak early diastolic PVF velocity in the RF group was significantly lower than that in the control, PN, and RS groups. The PVA was significantly greater in the PN group than in the other 3 groups. Further, the PVA in the RF and RS groups was significantly greater than that in the control group.

\section{Tissue Doppler Imaging Variables}

The peak early diastolic motion velocities at the mitral annulus and the LVPW were significantly lower in the RF, PN, and RS groups than in the control group (Table 3 ). 

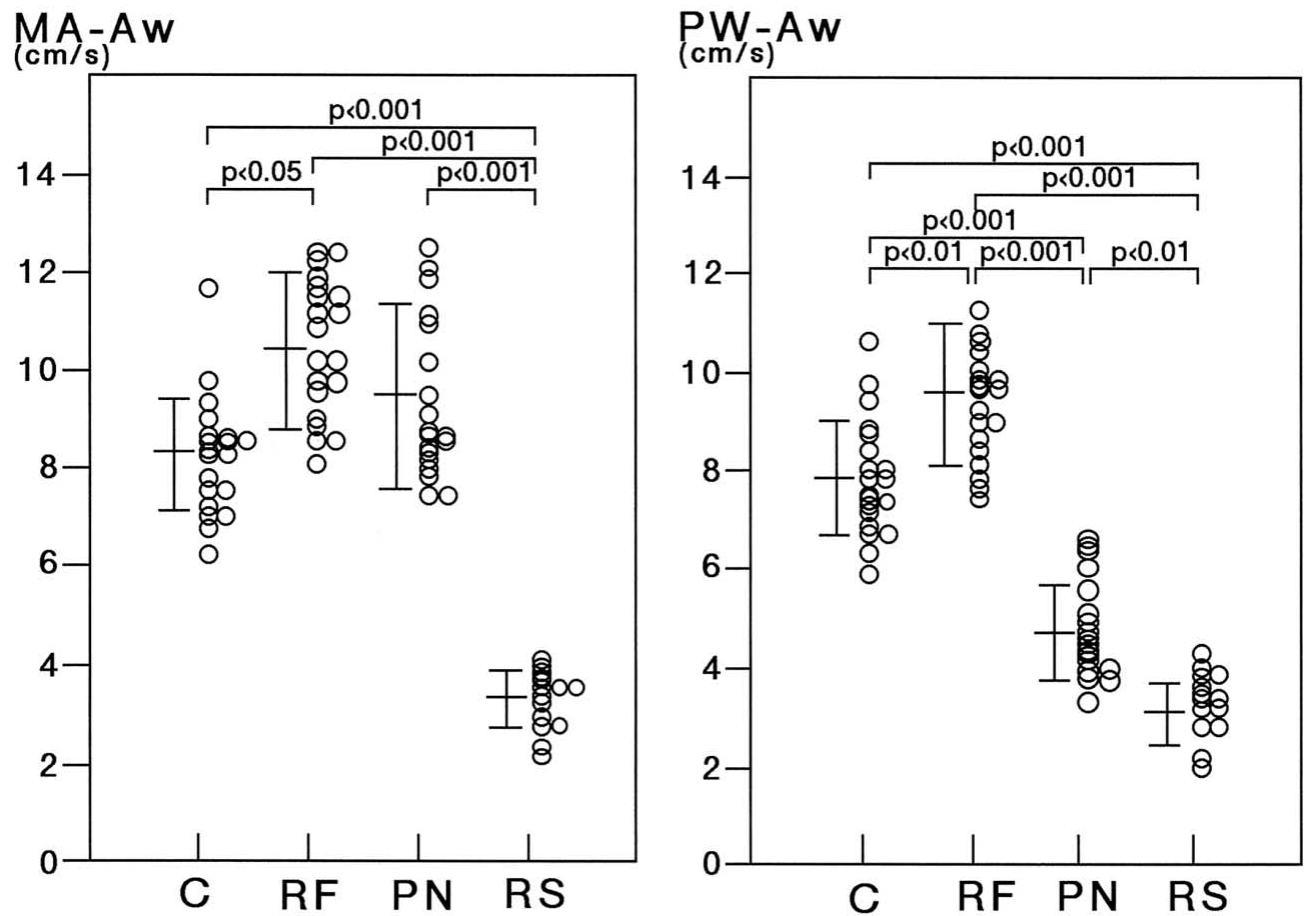

Fig 3. Comparisons of the peak atrial systolic motion velocities at the mitral annulus (MA-Aw) and posterior wall (PW$\mathrm{Aw}$ ) among control and patient groups. $\mathrm{C}$, control group; RF, relaxation failure group; PN, pseudonormalization group; RS, restriction group.

Table 1 Clinical Characteristics and M-Mode Echocardiographic Variables

\begin{tabular}{|c|c|c|c|c|c|c|c|c|}
\hline & $\begin{array}{c}\text { Age } \\
\text { (years) }\end{array}$ & $\begin{array}{c}H R \\
\text { (beats/min) }\end{array}$ & $\begin{array}{c}S B P \\
(m m H g)\end{array}$ & $\begin{array}{l}\angle A D \\
(\mathrm{~cm})\end{array}$ & $\begin{array}{c}L A-\% A C \\
(\%)\end{array}$ & $\begin{array}{l}L V D d \\
(\mathrm{~cm})\end{array}$ & $\begin{array}{c}m L V t h \\
(m m)\end{array}$ & $\begin{array}{c}\% F S \\
(\%)\end{array}$ \\
\hline$C(n=20)$ & $38 \pm 8$ & $72 \pm 9$ & $118 \pm 19$ & $3.3 \pm 0.3$ & $34 \pm 5$ & $4.7 \pm 0.3$ & $9 \pm 0.9$ & $38 \pm 5$ \\
\hline$R F(n=20)$ & $55 \pm 15 * *$ & $76 \pm 10$ & $111 \pm 34$ & $3.8 \pm 0.6 * * *$ & $22 \pm 6 * * *$ & $5.8 \pm 0.6^{* * *}$ & $9 \pm 1.1$ & $21 \pm 4 * * *$ \\
\hline$P N(n=19)$ & $57 \pm 16 * * *$ & $78 \pm 13$ & $106 \pm 31$ & $3.9 \pm 0.8 * * *$ & $19 \pm 7 * * *$ & $5.9 \pm 1.0 * * *$ & $11 \pm 1.2 * * * \dagger$ & $20 \pm 5 * * *$ \\
\hline$R S(n=14)$ & $53 \pm 17 * *$ & $80 \pm 15$ & $105 \pm 24$ & $4.0 \pm 1.1 * * *$ & $13 \pm 5 * * *+\hbar \S \S$ & $5.7 \pm 1.1 * * *$ & $11 \pm 1.3 * * *+\dagger$ & $20 \pm 4 * * *$ \\
\hline
\end{tabular}

** $p<0.01$, *** $p<0.001$ vs $N,{ }^{\dagger} p<0.001$ vs $R F,{ }^{s} p<<0.001$ vs $P N$.

$N$, control group; $R F$, relaxation failure group; $P N$, pseudonormalization group; $R S$, restriction group; HR, heart rate; SBP, systolic blood pressure; $L A D$, maximum left atrial dimension; $L A-\% A C$, percent fractional change of the left atrial area during atrial systole; LVDd, left ventricular end-diastolic dimension; $m L V t h$, mean left ventricular wall thickness at end-diastole; \%FS, percent fractional shortening of the left ventricle.

Table 2 Transmitral and Pulmonary Venous Flow Velocity Variables

\begin{tabular}{|c|c|c|c|c|c|c|c|c|}
\hline & $\begin{array}{c}E \\
(\mathrm{~cm} / \mathrm{s})\end{array}$ & $\begin{array}{c}A \\
(\mathrm{~cm} / \mathrm{s})\end{array}$ & $E / A$ & $\begin{array}{l}\text { IRT } \\
(\mathrm{ms})\end{array}$ & $\begin{array}{c}E-D T \\
(m s)\end{array}$ & $\begin{array}{c}P V S \\
(\mathrm{~cm} / \mathrm{s})\end{array}$ & $\begin{array}{c}P V D \\
(\mathrm{~cm} / \mathrm{s})\end{array}$ & $\begin{array}{c}P V A \\
(\mathrm{~cm} / \mathrm{s})\end{array}$ \\
\hline$C(n=20)$ & $74 \pm 9$ & $43 \pm 10$ & $1.8 \pm 0.4$ & $74 \pm 7$ & $145 \pm 26$ & $51 \pm 8$ & $60 \pm 9$ & $13 \pm 5$ \\
\hline$R F(n=20)$ & $50 \pm 11 * * *$ & $72 \pm 11 * * *$ & $0.7 \pm 0.2 * * *$ & $109 \pm 16^{* * *}$ & $198 \pm 32 * * *$ & $58 \pm 15$ & $33 \pm 14 * *$ & $24 \pm 9 * * *$ \\
\hline$P N(n=19)$ & $84 \pm 20^{\dagger \dagger}$ & $49 \pm 11^{\dagger \dagger}$ & $1.7 \pm 0.4^{\dagger \dagger}$ & $72 \pm 1^{\dagger \dagger}$ & $140 \pm 18^{\dagger \dagger}$ & $31 \pm 11$ ***十⿱亠巾十力口 & $63 \pm 19^{\dagger \dagger}$ & $37 \pm 13 * * *$ \\
\hline$R S(n=14)$ & $87 \pm 13^{t \dagger}$ & $32 \pm 11 *+7 \S \S$ & $2.8 \pm 0.4 * * *+\dagger \S \S$ & $72 \pm 9$ & $110 \pm 23^{* *+t \dagger}$ & $20 \pm 11 * * * \dot{t}$ & $61 \pm 19^{\prime \dagger}$ & $20 \pm 7 * \S \S$ \\
\hline
\end{tabular}

$* p<0.05, * * p<0.01, * * * p<0.001$ vs $N,{ }^{\dagger} p<0.001$ vs $R F, s_{s} p<0.001$ vs $P N$.

E, peak early diastolic transmitral flow velocity; A, peak atrial systolic transmitral flow velocity; E/A, ratio of peak early diastolic to atrial systolic transmitral flow velocity; IRT, isovolumic relaxation time; E-DT, deceleration time of early diastolic transmitral flow; PVS, peak second systolic pulmonary venous flow velocity; PVD, peak early diastolic pulmonary venous flow velocity; PVA, peak atrial systolic pulmonary venous flow velocity.

However, there were no significant differences in the peak early diastolic motion velocities among the 3 disease groups. The Aw at the mitral annulus was significantly lower in the RS group than in the other 3 groups (Fig 3, left), and was significantly greater in the RF group than in the control group. Comparison of the Aw at the LVPW demonstrated that $\mathrm{RF}>$ control $>\mathrm{PN}>\mathrm{RS}$ (Fig 3, right). There were no significant differences in the Aw between the mitral annulus and the LVPW in the control and RS groups. In the RF and PN groups, however, the Aw at the mitral annulus was significantly greater than that at the LVPW.

\section{Hemodynamic Variables}

The mPCWP was significantly greater in the 3 disease groups than in the control group (Table 4; Fig 4, left). The 

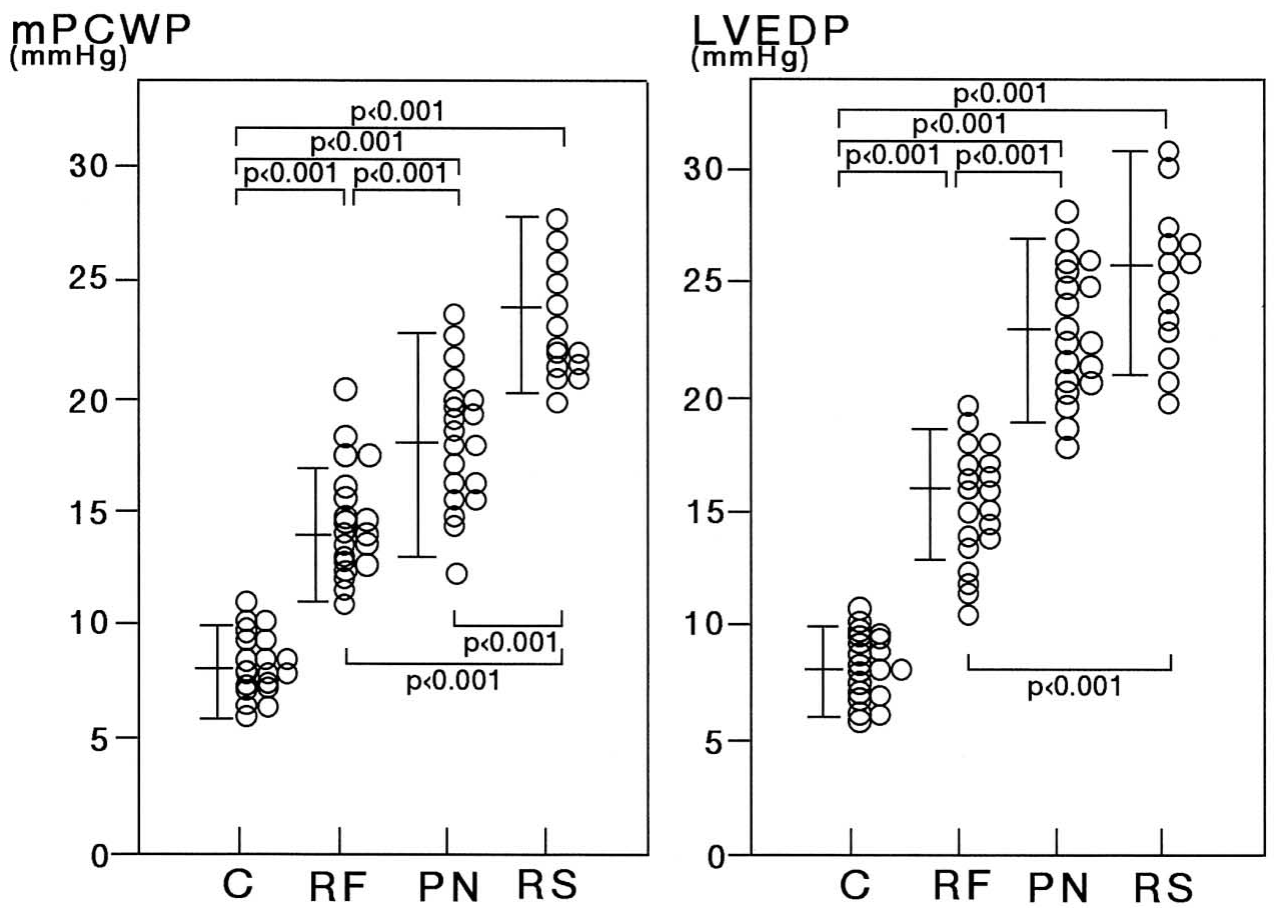

Fig 4. Comparisons of the mean pulmonary capillary wedge pressure (mPCWP) and left ventricular end-diastolic pressure (LVEDP) among control and patient groups. C, control group; RF, relaxation failure group; PN, pseudonormalization group; RS, restriction group.

Table 3 Tissue Doppler Imaging Variables

\begin{tabular}{|c|c|c|c|c|}
\hline & $\begin{array}{c}M A-E w \\
(\mathrm{~cm} / \mathrm{s})\end{array}$ & $\begin{array}{c}P W-E w \\
(\mathrm{~cm} / \mathrm{s})\end{array}$ & $\begin{array}{c}M A-A w \\
(\mathrm{~cm} / \mathrm{s})\end{array}$ & $\begin{array}{c}P W-A w \\
(\mathrm{~cm} / \mathrm{s})\end{array}$ \\
\hline$C(n=20)$ & $17.3 \pm 3.4$ & $16.2 \pm 3.4$ & $8.3 \pm 1.1$ & $7.9 \pm 1.2$ \\
\hline$R F(n=20)$ & $9.3 \pm 2.2 * * *$ & $8.7 \pm 2.9 * * *$ & $10.5 \pm 1.6^{*}$ & $9.7 \pm 1.5^{* * \pi /}$ \\
\hline$P N(n=19)$ & $8.5 \pm 3.0 * * *$ & $8.3 \pm 3.2 * * *$ & $9.5 \pm 1.9$ & $4.7 \pm 1.0 * * *++\pi \%$ \\
\hline$R S(n=14)$ & $8.4 \pm 2.9 * * *$ & $7.7 \pm 3.3 * * *$ & $3.4 \pm 0.6^{* * *+\dagger \S \S}$ & $3.2 \pm 0.6 * * *+i \xi$ \\
\hline
\end{tabular}

${ }^{*} p<0.05, * * p<0.01, * * * p<0.001$ vs $N,{ }^{\dagger} p<0.001$ vs $R F,{ }^{*} p<0.01,{ }^{s} s_{p}<$ 0.001 vs $P N$, " $p<0.05$, "II $p<0.001$ vs MA.

MA, mitral annulus; $P W$, posterior wall; Ew, peak early diastolic wall motion velocity; Aw, peak atrial systolic wall motion velocity.

mPCWP was significantly greater in the PN and RS groups than in the RF group, and was significantly greater in the RS group than in the PN group. The LVEDP was significantly greater in the 3 disease groups than in the control group, and was significantly greater in the PN and RS groups than in the RF group (Fig 4, right). However, there was no significant difference in the LVEDP between the $\mathrm{PN}$ and RS groups.

TMF, PVF, and mitral annulus and LVPW motion velocity patterns in 4 representative patients from each group are shown in Fig 5.

\section{Discussion}

\section{$T M F$ and $P V F$}

It is very important to evaluate the $\mathrm{LV}$ diastolic function noninvasively. At present, the TMF velocity is used most frequently for this purpose. Furthermore, detailed information concerning the hemodynamic relationship between the LA and LV can be obtained by evaluating the PVF velocity together with the TMF velocity!-9 Oki et al have measured the PVF velocity using transesophageal pulsed Doppler
Table 4 Hemodynamic Variables

\begin{tabular}{rccc}
\hline \hline & $\begin{array}{c}\text { LVEF } \\
(\%)\end{array}$ & $\begin{array}{c}m P C W P \\
(m m H g)\end{array}$ & $\begin{array}{c}\text { LVEDP } \\
(m m H g)\end{array}$ \\
\hline$C(n=20)$ & $68 \pm 10$ & $8 \pm 2$ & $8 \pm 2$ \\
$R F(n=20)$ & $36 \pm 5 * * *$ & $14 \pm 3 * * *$ & $16 \pm 3 * * *$ \\
$P N(n=19)$ & $35 \pm 4 * * *$ & $18 \pm 4 * * * \dagger$ & $23 \pm 4 * * * \dagger$ \\
$R S(n=14)$ & $34 \pm 4 * * *$ & $24 \pm 4 * * *+\xi \xi$ & $26 \pm 5 * * * \dagger ;$
\end{tabular}

*** $p<0.001$ vs $N,{ }^{\dagger} p<0.001$ vs $R F, s_{s} p<0.001$ vs $P N$.

$L V E F$, left ventricular ejection fraction; $m P C W P$, mean pulmonary

capillary wedge pressure; LVEDP, left ventricular end-diastolic pressure.

echocardiography in patients with dilated failing heart and a PN pattern of the TMF velocity? They further demonstrated that the PVA increases in patients with no increase in the MPCWP corresponding to the markedly elevated LVEDP, whereas the PVA is normal or decreases in patients with both elevated MPCWP and LVEDP. If the LVEDP is elevated markedly, blood in the LA flows toward the pulmonary veins rather than toward the LV during atrial systole because the pulmonary venous system has a lower resistance during ventricular diastole. This causes the PVA to be greater than the peak atrial systolic TMF velocity (PN group). However, as pulmonary congestion increases, the LA falls into an afterload mismatch, resulting in decreases in peak atrial systolic velocities for both the TMF and PVF (RS group). However, these parameters generally are influenced by loading conditions, especially LA pressure. Accordingly, it has been recognized that parameters obtained from TMF and PVF velocities do not truly reflect abnormalities in LV diastolic function? ${ }^{3-9}$

\section{Mitral Annular and LVPW Motion Velocities}

It has been reported that the early diastolic parameters 


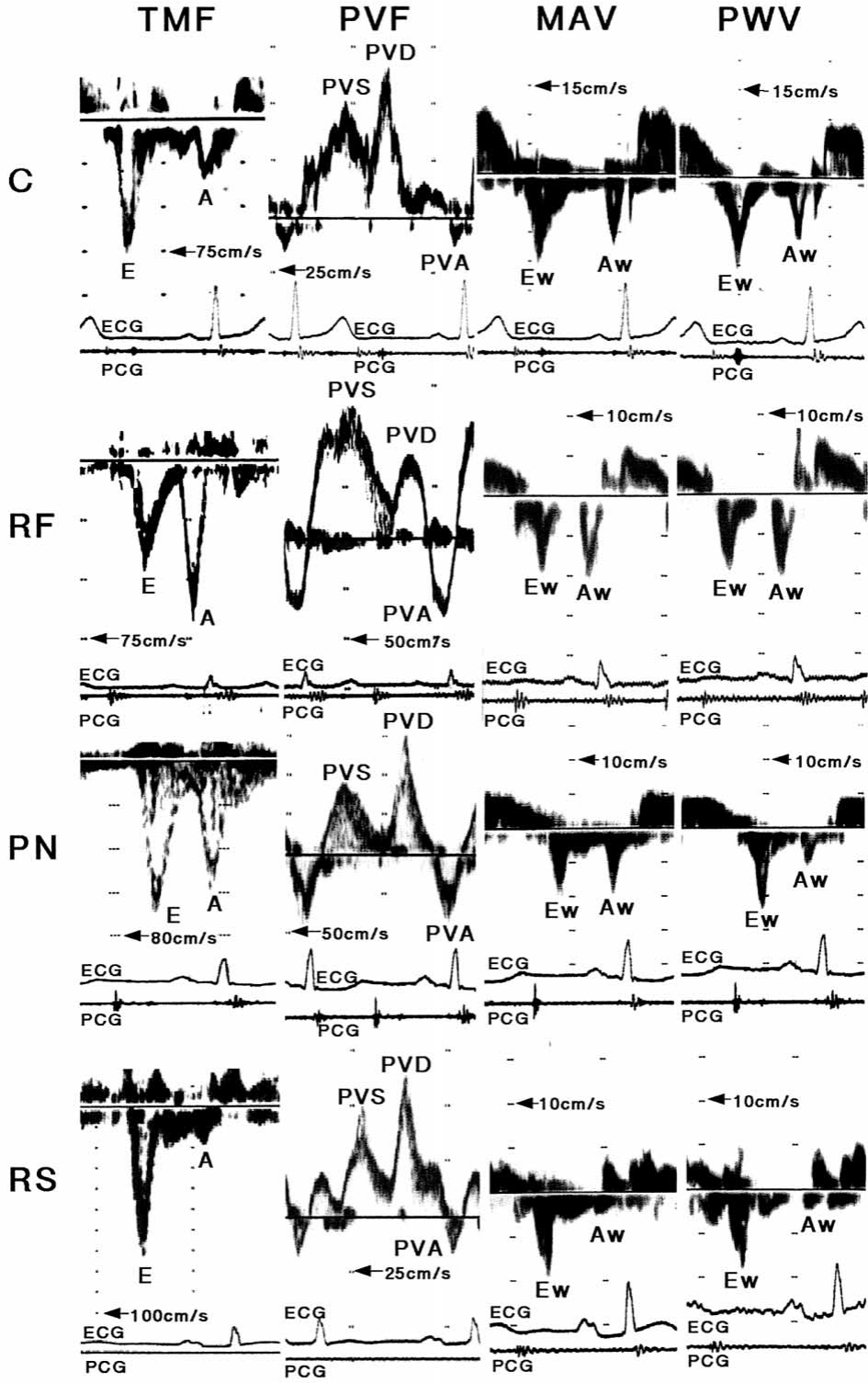

Fig 5. Transmitral flow (TMF), pulmonary venous flow (PVF), and wall motion velocities at the mitral annulus of the posterior wall site (MAV) and at the left ventricular posterior wall (PWV) in a representative normal subject (C), and 3 representative patients with relaxation failure (RF:E/A $\leq 1)$, pseudonormalization (PN:E/A $>1$ ) and restriction (RS:E/A $\geq 2$ ) patterns of the TMF. The peak atrial systolic motion velocity (Aw) at the posterior wall is markedly decreased in both the patients with PN and RS patterns. In contrast, the patient with PN pattern demonstrates a marked increase in Aw at the mitral annulus compared with the value from the patient with RS pattern. Ew, peak early diastolic wall motion velocity; E, peak early diastolic velocity of TMF; A, peak atrial systolic velocity of TMF; ECG, electrocardiogram; PCG, phonocardiogram. derived from the motion velocity of the LVPW along the short axis correlate closely with the time constant of LV pressure decay during isovolumic diastole ${ }^{22}$ Nagueh et al have reported that a good correlation was observed between the ratio of the early diastolic TMF velocity to the early diastolic wall motion velocity at the mitral annulus along the long axis and the mPCWP23 Moreover, Sohn et al have emphasized that the wall motion velocity at the mitral annulus along the long axis is relatively preload independent 24

Alam et al have reported that the amplitude of mitral annular motion during atrial systole determined by $\mathrm{M}$ mode echocardiography has a significant positive correlation with the atrial systolic TMF velocity 12 The mitral annular motion velocity obtained by pulsed TDI consists of 2 peaks during early diastole and atrial systole. Further, atrial systolic motion velocity (Aw) increases in patients with LV hypertrophy and an $\mathrm{E} / \mathrm{A}<125$ In the present study, the Aws at the LV posterior wall and mitral annulus were both significantly greater in the RF group than in the control group, which is consistent with that reported by Rodriguez et al25
The atrial systolic waves from the mitral annular motion velocity patterns is believed to be influenced by abnormal hemodynamic events occurring not only on the LV side but also on the LA side!1,12,19,20 There have been few reports evaluating the differences between mitral annular and LV wall motion velocity patterns using pulsed TDI in various disease conditions ${ }^{22-27}$ Rychik et al have reported that the Aw at the mitral annulus differs from that at the LVPW in healthy children, and that mitral annular motion is influenced not only by LV systolic function but also by LA function. ${ }^{19}$ However, they evaluated the Aw at the LVPW along the short axis and the Aw at the mitral annulus along the long axis. In the present study, we recorded the motion velocity patterns for both the mitral annulus and the LVPW along the long axis. The Aw at the LVPW was markedly lower in the PN and RS groups than in the RF and control groups. Moreover, the RS group demonstrated marked decreases in Aw at the mitral annulus, corresponding to the decreases in PVA, compared with the value obtained from the PN group. 


\section{Clinical Significance}

It has recently been reported that the prognosis of patients with LV systolic failure can be determined by the TMF velocity patterns ${ }^{28,29}$ However, it is difficult to evaluate the hemodynamic relationship between the LA and pulmonary venous system from the TMF velocity variables alone. In the present study, we evaluated difference in Aws between the mitral annulus and the LVPW along the long axis using transthoracic pulsed TDI. As a result, we demonstrated that a marked decrease in Aw at the mitral annulus in the RS group results from significant decreases in functional reserve of the pulmonary venous system (PVA) and LA pump function (LA-\%AC), and a significant elevation in LA pressure (mPCWP) compared with the PN group. Therefore, the Aws at the mitral annulus and the LVPW along the long axis determined by pulsed TDI can be used to understand the hemodynamic relationship between the LA and LV during atrial systole in the presence of an elevated LVEDP and LV failure. Furthermore, this examination offers the advantage that it is noninvasive and easy.

\section{Study Limitations}

Patients demonstrating regional LV asynergy were excluded from the present study. In such patients, wall motion velocities at the mitral annulus and LVPW can differ ${ }^{27,30}$ LA atrium and the LV cannot be evaluated by assessing only the regional mitral annular or LV wall motion velocity. Moreover, differentiation between patients with LA myocardial failure and those with LA afterload mismatch by evaluating the TMF and PVF velocities is limited? It remains to be determined whether the parameters obtained from pulsed TDI in the present study are useful in differentiating both these abnormal conditions.

\section{References}

1. Appleton CP, Hatle LK, Popp RL: Relation of transmitral flow velocity patterns to left ventricular diastolic function: new insights from a combined hemodynamic and Doppler echocardiographic study. J Am Coll Cardiol 1988; 12: 426-440

2. Nishimura RA, Tajik AJ: Evaluation of diastolic filling of the left ventricle in health and disease: Doppler echocardiography is the clinician's Rosetta Stone. J Am Coll Cardiol 1997; 30: 8-18

3. Choong CY, Herrmann HC, Weyman AE, Fifer MA: Preload dependence of Doppler-derived indexes of left ventricular diastolic function in humans. J Am Coll Cardiol 1987; 10: 800-808

4. Courtois M, Vered Z, Barzilai B, Ricciotti NA, Perez JE, Ludbrook PA: The transmitral pressure-flow velocity relation: effect of abrupt preload reduction. Circulation 1988; 78: 1459-1468

5. Appleton CP, Hatle LK, Ropp RL: Demonstration of restrictive ventricular physiology by Doppler echocardiography. J Am Coll Cardiol 1988; 11: 757-768

6. Thomas JD, Choong CY, Flachskampf FA, Weyman AE: Analysis of the early transmitral Doppler velocity curve: effect of primary physiologic changes and compensatory preload adjustment. $J \mathrm{Am}$ Coll Cardiol 1990; 16: 644-655

7. Nishimura RA, Abel MD, Hatle LK, Tajik AJ: Relation of pulmonary vein to mitral flow velocities by transesophageal Doppler echocardiography: effect of different loading conditions. Circulation 1990; 81: $1488-1497$

8. Oki T, Fukuda N, Iuchi A, Tabata T, Yamada H, Manabe K, et al: Transesophageal pulsed Doppler echocardiographic evaluation of left atrial systolic performance in hypertrophic cardiomyopathy: combined analysis of transmitral and pulmonary venous flow velocities. Clin Cardiol 1997; 20: 47-54

9. Oki T, Fukuda N, Iuchi A, Tabata T, Tanimoto M, Manabe K, et al: Left atrial systolic performance in the presence of elevated left ven- tricular end-diastolic pressure: evaluation by transesophageal pulsed Doppler echocardiography of left ventricular inflow and pulmonary venous flow velocities. Echocardiography 1996; 13: 23-32

10. Keren G, Sonnenblick EH, Lejemtel TH: Mitral annulus motion: Relation to pulmonary venous and transmitral flows in normal subjects and in patients with dilated cardiomyopathy. Circulation 1988; 78: $621-629$

11. Jones CJH, Song GJ, Gibson DG: An echocardiographic assessment of atrial mechanical behaviour. Br Heart J 1991; 65: 31-36

12. Alam M, Hoglund C: Assessment by echocardiogram of left ventricular diastolic function in healthy subjects using the atrioventricular plane displacement. Am J Cardiol 1992; 69: 565-568

13. Blomstrand P, Kongstad O, Broqvist M, Dahlstrom U, Wranne B: Assessment of left ventricular diastolic function from mitral annulus motion, a comparison with pulsed Doppler measurements in patients with heart failure. Clin Physiol 1996; 16: 483-493

14. Isaaz K, Thompson A, Ethevenot G, Cloez JL, Brembilla B, Pernot C: Doppler echocardiographic measurement of low velocity motion of the left ventricular posterior wall. Am J Cardiol 1989; 64: 66-75

15. McDicken WN, Sutherland GR, Moran CM, Gordon LN: Color Doppler velocity imaging of the myocardium. Ultrasound Med Biol 1992; 18: 651-654

16. Yamazaki N, Mine Y, Sano A, Hirama M, Miyatake K, Yamagishi $\mathrm{M}$, et al: Analysis of ventricular wall motion using color-coded tissue Doppler imaging system. Jpn J Appl Physiol 1994; 33: 3141-3146

17. Palka P, Lange A, Fleming AD, Sutherland GR, Fenn LN, McDicken WN: Doppler tissue imaging: myocardial wall motion velocities in normal subjects. J Am Soc Echocardiogr 1995; 8: 659-668

18. Garcia MJ, Rodriguez L, Ares M, Griffin BP, Klein AL, Stewart WJ, et al: Myocardial wall velocity assessment by pulsed Doppler tissue imaging: characteristic findings in normal subjects. Am Heart $J$ 1996; 132: 648-656

19. Rychik J, Tian Z: Quantitative assessment of myocardial tissue velocities in normal children with Doppler tissue imaging. Am J Cardiol 1996; 77: 1254-1257

20. Tanimoto T, Pai RG: Effect of isolated left atrial enlargement on mitral annular size and valve competence. Am J Cardiol 1996; 77: $769-774$

21. Burch GE, Giles TD, Colcolough HL: Ischemic cardiomyopathy. Am Heart J 1970; 79: 291-292

22. Oki T, Tabata T, Yamada H, Wakatsuki T, Shinohara H, Nishikado A, et al: Clinical application of pulsed Doppler tissue imaging for assessing abnormal left ventricular relaxation. Am J Cardiol 1997; 79: $921-928$

23. Nagueh SF, Middleton KJ, Kopelen HA, Zoghbi WA, Quinones MA: Doppler tissue imaging: a noninvasive technique for evaluation of left ventricular relaxation and estimation of filling pressures. $J \mathrm{Am}$ Coll Cardiol 1997; 30: 1527-1533

24. Sohn D, Chai I, Lee D, Kim H, Kim H, Oh B, et al: Assessment of mitral annulus velocity by Doppler tissue imaging in the evaluation of left ventricular diastolic function. J Am Coll Cardiol 1997; 30: $474-480$

25. Rodriguez L, Garcia MJ, Ares M, Griffin BP, Nakatani S, Thomas JD: Assessment of mitral dynamics during diastole by Doppler tissue imaging: comparison with mitral Doppler inflow in subjects without heart disease and in patients with left ventricular hypertrophy. $\mathrm{Am}$ Heart J 1996; 131: 982-987

26. Garcia MJ, Rodriguez L, Ares M, Griffin BP, Thomas JD, Klein AL: Differentiation of constrictive pericarditis from restrictive cardiomyopathy: assessment of left ventricular diastolic velocities in longitudinal axis by Doppler tissue imaging. J Am Coll Cardiol 1996; 27: $108-114$

27. Gulati VK, Katz WE, Follansbee WP, Gorcsan J III: Mitral annular descent velocity by tissue Doppler echocardiography as an index of global left ventricular function. Am J Cardiol 1996; 77: 979-984

28. Rihal CS, Nishimura RA, Hatle LK, Bailey KR, Tajik AJ: Systolic and diastolic dysfunction in patients with clinical diagnosis of dilated cardiomyopathy: relation to symptoms and prognosis. Circulation 1994; 90: $2772-2779$

29. Xie G, Berk MR, Smith MD, Curley JC, DeMaria AN: Prognostic value of Doppler transmitral flow pattern in patients with congestive heart failure. J Am Coll Cardiol 1994; 24: 132-139

30. Fukuda K, Oki T, Tabata T, Iuchi A, Ito S: Regional left ventricular wall motion abnormalities in myocardial infarction and mitral annular descent velocities studied with pulsed tissue Doppler imaging. J Am Soc Echocardiogr 1998; 11: 841-848 